\title{
FETOMATERNAL OUTCOME OF PREGNANCY BETWEEN 40 AND 42 WEEKS
}

\author{
DR. NAZIA AMJAD \\ Department of Obs \& Gynae \\ Ittefaq Hospital (Trust) Lahore \\ DR. TAYYBA IMRAN \\ Department of Obs \& Gynae \\ Ittefaq Hospital (Trust) Lahore
}

DR. AHSEN NAZIR AHMED

Accident and Emergency Jinnah Hospital, Lahore.

\begin{abstract}
Objective: To evaluate whether serial monitoring of fetuses beyond 40 weeks with biophysical profile and non stress test improves the fetal outcome in terms of morbidity and mortality.Design: Prospective control study. Setting: Department of Obstetrics \& Gynaecology at Ittefaq Hospital( Trust) Lahore,. Period: From Jan, 2007 to June 2008.Patients and Methods: Two hundred women with singleton uncomplicated pregnancies at 40 weeks were divided into two groups; A study group with biweekly biophysical profile and non stress test and a control group with biweekly antenatal clinical assessment with fetal kick count chart. Main outcome measures were onset of labour, mode of delivery, Apgar score, presence of meconium and admission to nursery. Results: The age of patients ranged between 18 to 39 years with the mean of 26.33. The range of gravidity was between primigravida to gravida seven. Ninety six percent of total patients were delivered by 41 weeks and 6 days. $18 \%$ of women were induced in study group compared to $11 \%$ in control group ( $p$ value $=0.124$ ). Cesarean section rate was $8 \%$ in study group and $11 \%$ in control group. Rate of instrumental deliveries was $6.5 \%$ in study group while $11.2 \%$ in control group. The difference in mode of delivery was found to be insignificant $(p=0.538)$.Weights of the babies ranged between 2.6 to $4.4 \mathrm{~kg}$ with a mean of $3.246 \mathrm{~kg}$. Cumulative \%age of APGAR score at 5 minutes was more than $6 / 10$ in $92.5 \%$ cases ( $p=0.665$ ).Meconium was found in $18 \%$ of cases in study group and $22 \%$ of control group $(p=0.917) .12 \%$ of the babies were admitted to nursery in study group compared to $16 \%$ in control group. Perinatal mortality was found to be $10 / 1000$ in control group while there was no perinatal death in study group. Conclusions: The difference of outcome between two groups was found to be statistically insignificant which concluded the validity of either mode of management.
\end{abstract}

Key words: $\quad$ Prolonged pregnancy, Perinatal morbidity and mortality, fetal monitoring, biophysical profile.

\section{INTRODUCTION}

Pregnancy at term is a period of five weeks from 37 to 42 weeks i-e 260 to 294 days. Prolonged pregnancy is defined by the International Federation of Gynaecologists and Obstetricians (FIGO) as any pregnancy which exceeds 294 days from the first day of last menstrual period. Approximately $7.5 \%$ of pregnancies continue 42 weeks or beyond ${ }^{1}$. The period after 40 weeks is of utmost 
concern for the patient as well as medical attendant. Tests of fetal well being are usually carried out after 41 weeks because unexpected morbidity and mortality can occur after this period ${ }^{2}$. However, early ultrasound scanning and induction of labour at 41 weeks would add considerable financial burden to the system ${ }^{3}$.

Fetal risks of prolonged pregnancy include fetal asphyxia due to decrease in the delivery of the required amount of oxygen to the fetus as there is decline in the placental functions. There is 4 to 5 times increased risk of perinatal mortality when pregnancies with compromised placental functions prior to term are allowed to continue postdate. Clinical manifestations of asphyxia may range from presence of variable decelerations to development of permanent neurological sequelae. There is also decrease in the amount of amniotic fluid which in turn is associated with fetal distress due to umbilical cord compression. The passage of meconium is seen in about $19 \%$ of deliveries at 40 weeks. Its incidence is 27 to $42 \%$ in pregnancies at or beyond 42 weeks. Meconium aspiration was found in $0.6 \%$ of infants at 40 weeks and in $1.6 \%$ at 42 weeks which is a major contributory factor towards perinatal morbidity and mortality as it can lead to pneumonia ,pneumothorax and development of pulmonary hypertension. Combination of fetal distress and meconium release are 3 times more in deliveries at or after 42 weeks. Studies have shown that there is increased risk of perinatal mortality after 42 weeks ${ }^{4,5}$ and thereafter which is twice as compared to perinatal mortality at 40 weeks. After 38 weeks the fetus grows but at a reduced velocity and becomes macrosomic. Its incidence at 42 weeks is $25 \%$. Fetal macrosomia is associated with shoulder dystocia and neonatal complications like hypoglycemia, jaundice and temperature instability. Incidence of shoulder dystocia is $18 \%$ at 42 weeks.

Other fetal risks include postmaturity syndrome ( risk is $10 \%$ ) and some long term sequalae as the mental scores of post term infants were lower and there were quantifiable delays of either mental or physical development.

Maternal risks include increase in maternal morbidity due to anxiety, prolonged labour, instrumental delivery and Cesarean section(1.3\% at 42 weeks ) and birth canal trauma due to fetal macrosomia. There is also increased risk of epilepsy in the neonate particularly if delivered by instrumental delivery or cesarean section ${ }^{6}$. Because of these risks, some authoritative organizations recommend that women should be offered induction of labour after 41 weeks gestation ${ }^{7}$.

Aim of the study was to evaluate whether serial monitoring of fetuses beyond 40 weeks with biophysical profile and non stress test can improve the fetal outcome. Results of the study helped in making a definitive assessment and management plan for pregnancy beyond 40 weeks to reduce the risk of fetal and neonatal morbidity.

\section{PATIENTS AND METHODS}

Two Hundred pregnant women were selected from antenatal outpatient irrespective of their age, parity and socioeconomic class. They were all booked with regular menstrual cycles, were sure of last menstrual period and had completed 40 weeks, were not taking any hormonal preparation for contraception prior to pregnancy, which had no maternal or fetal risks of prolongation of pregnancy beyond 40 weeks and had no contraindication or vaginal delivery.

The patients excluded from study were those who had some maternal or obstetrical complications ( Medical disorders, antepartum haemorrhage etc), with multifetal pregnancy and with congenitally abnormal fetus.

At 40 weeks, patients were counseled, informed consent was taken and a detailed antenatal checkup was performed. These patients were randomly divided into 2 groups.

In study group, fetal monitoring was done with biweekly biophysical profile and non stress test. When results were found to be normal, spontaneous onset of labour was awaited till 42 weeks. However, in cases of fetal compromise at any stage, termination of pregnancy was planned.

In control group, patients were called biweekly for 
antenatal checkup. Clinical assessment of amount of amniotic fluid and enquiry about fetal kick count was done. With normal kick count, spontaneous onset of labour was awaited. However, in cases of sluggish fetal movements, termination of pregnancy was planned.

All patients who had not gone into spontaneous labour were induced at 42 weeks.

Results of the study were analyzed statistically by using Chi Square test. A p value of less than 0.05 was considered significant.

\section{RESULTS}

During this one and half year study, a total of 200 patients were included. The age of the patients ranged between 18 to 39 years with a mean of 25.77 years. Most of the patients were between 26 to 30 years of age i-e $48 \%$ in study group and $52 \%$ in control group.

The range of gravidity was between primigravida to gravida seven with $33 \%$ of primigravidae in study group and $36 \%$ in control group.

Only 8 patients ( $4 \%$ ) out of 200 reached up to 42 weeks o gestation, 4 in each group. Out of these 8,3 went into spontaneous labour while induction was carried out in 5 patients. Four out of 8 had spontaneous vaginal delivery, 2 had instrumental vaginal delivery and had emergency section due to fetal distress. Five out of 8 patients had moderate to thick meconium in liquor. Up to 41 weeks and 6 days, $96 \%$ of women in both groups were either delivered spontaneously or induced due to some indication.

Spontaneous onset of labour was found in $80 \% 0$ patients in control group and in $89 \%$ patients in study group. $(p$ value $=0.124)$. Two patients in study had neither gone into spontaneous labour nor were induced but had emergency cesarean section due to fetal distress diagnosed by poor biophysical profile.

In the study group, a major indication of induction was poor biophysical profile i-e in $56 \%$ of inductions while $33 \%$ o inductions were due to premature ruptures of membranes. Only $11 \%$ were due to completed 42 weeks. In the control group, $45.4 \%$ of inductions were due to premature rupture of membranes and $27.3 \%$ each for sluggish fetal movements and completed 42 weeks.

The cesarean section rate in study group was $8 \%$.In control group, it was $11 \%$ ( $p$ value $=0.538$ )

\begin{tabular}{|c|c|c|}
\hline \multirow[t]{2}{*}{ Mode of deliver } & \multicolumn{2}{|c|}{ No of cases } \\
\hline & Study group & Control Group \\
\hline $\begin{array}{l}\text { Spontaneous } \\
\text { vaginal delivery }\end{array}$ & 86 & 79 \\
\hline Vacuum extraction & 2 & 5 \\
\hline Forceps deliver & 4 & 5 \\
\hline $\begin{array}{l}\text { Emergency } \\
\text { cesarean section }\end{array}$ & 8 & 11 \\
\hline Total & 100 & 100 \\
\hline
\end{tabular}

Fetal distress was found to be the most common indication of instrumental delivery as well as Cesarean section ie in 4 patients ( $50 \%$ ) in study group and $7(64 \%$ )in control group).Failure to progress was the indication in 3 cases $(37.5 \%$ ) in study group and 2 cases (18\%) in control group, while failure of induction was found in one case each in study group (12.5\%) and control group $(9 \%)$.

Rate of instrumental vaginal deliveries was found to be $6 \%$ in study group. Indications were fetal distress in 3 cases $(50 \%)$ and failure to progress in other 3 cases $(50 \%)$.In the control group,10 patients had instrumental vaginal deliveries, 6 for fetal distress $(60 \%)$ and 4 for failure to progress $(40 \%)$.

Only 3 babies out of 200 had a birth weight of more than $4 \mathrm{~kg}$, none weighed more than $4.5 \mathrm{~kg}$. Mean birth weight in study group was $3.247 \mathrm{~kg}$ and 3.24 in control group. Only one out of 200 babies had a 3/10 APGAR score at 5 minutes. $87 \%$ babies in study group and $85 \%$ in control group were born with APGAR score of 8/10 or more. The 
difference in the APGAR score between both groups was statistically insignificant ( $p$ value $=0.665$ ).

\begin{tabular}{|l|c|c|}
\hline \multirow{2}{*}{ Table-ll. Apgar score of babies born at 5 minutes. } \\
\hline \multirow{2}{*}{ Apgar score at 5 minutes } & \multicolumn{2}{|c|}{ No of cases } \\
\cline { 2 - 3 } & Study group & Control group \\
\hline $8 / 10$ and above & 87 & 85 \\
\hline $6 / 10$ and $7 / 10$ & 6 & 8 \\
\hline $4 / 10$ and $5 / 10$ & 7 & 6 \\
\hline Less than $4 / 10$ & - & 1 \\
\hline Total & 100 & 100 \\
\hline
\end{tabular}

\begin{tabular}{|l|c|c|}
\hline \multicolumn{2}{|c|}{$\begin{array}{c}\text { Table-III. Presence of meconium in liquor in deliveries } \\
\text { between } \mathbf{4 0}-\mathbf{4 2} \text { weeks. }\end{array}$} \\
\hline Colour of Liquor & \multicolumn{2}{|c|}{ No of cases } \\
\hline & Study group & Control group \\
\hline Clear liquor & 82 & 78 \\
\hline $\begin{array}{l}\text { Meconium tinged } \\
\text { liquor }\end{array}$ & 6 & 7 \\
\hline $\begin{array}{l}\text { Meconium stained } \\
\text { liquor }\end{array}$ & 4 & 5 \\
\hline $\begin{array}{l}\text { Thick paste like } \\
\text { meconium }\end{array}$ & 8 & 10 \\
\hline Total & 100 & 100 \\
\hline
\end{tabular}

Meconium stained liquor was found in $18 \%$ cases in study group and in $22 \%$ patient in control group ( $p$ value+ 0.917).Although the meconium was found in the trachea of $50 \%$ babies, meconium aspiration syndrome developed in only one baby of control group. This was the only perinatal death.

Twelve babies in study group and 16 in control group were shifted to newborn nursery for further evaluation. Four babies in study group and 6 in control group were admitted for septic workup due to history of premature rupture of membranes. ${ }^{*}$ in study group and 10 in control group were admitted due to thick meconium.

\begin{tabular}{|l|c|}
\hline \multicolumn{2}{|c|}{ Table-IV. Admission to newborn nursery. } \\
\hline Groups & No of admissions \\
\hline Study group $(n=100)$ & 12 \\
\hline Control group $(n=100)$ & 16 \\
\hline
\end{tabular}

\section{DISCUSSION}

Available data has shown that when calculated from the first day of last menstrual period, about $90 \%$ of patients deliver before 42 weeks while $10 \%$ pregnancies continue beyond 42 weeks $^{8}$.It is evaluated that 20 to $45 \%$ women cannot remember the exact date of last menstrual period $^{9}$. Assessment of gestational age in early pregnancy reduces the incidence of prolonged pregnancy by $1 / 2$ to $2 / 3$. Where possible, first trimester ultrasound rather than last menstrual period should be relied upon to assess pregnancy duration ${ }^{10}$. Reuss et al found that incidence of prolonged pregnancy was reduced to $3 \%$ when gestational age was calculated using ultrasound criteria. $97 \%$ of the patients were delivered before 42 weeks of gestation ${ }^{11}$.

These results are comparable with this study in which $96 \%$ of the patients were delivered by 41 weeks and 6 days. Only 8 patients reached 42 weeks out of whom 3 went into spontaneous labour and 5 were induced in whom the pregnancy was likely to continue beyond 42 weeks.

The other factor responsible for reduction in the number of postdated pregnancies is induction of labour. In this study,24 out of 200 patients were induced before 42 weeks or different maternal or fetal indications. True incidence of prolonged pregnancy is also reduced by induction of labour earlier due to other reasons like maternal request and pregnancy related complications ${ }^{12}$.

Incidence of casarean section is 10 to $25 \%$ of total deliveries. Studies show that the rate of cesarean section is higher in expectantly managed group after 41 weeks when compared with induction of labour. In women undergoing induction of labour, cervical length and parity provides significant independent prediction of likelihood of cesarean section ${ }^{13}$. Risk factors intrinsic to 
patient rather than induction of labour are the cause of excess operative delivery in women with prolonged pregnancy $^{14}$. Cesarean section rate is higher due to fetal distress ${ }^{15}$. These results are comparable to this study in which overall cesarean section rate was $9.5 \%$. Also, Fetal distress was major indication for the operative delivery i-e $50 \%$ in study group and $65 \%$ in control group.

The incidence of meconium stained liquor is more in prolonged pregnancies. Its incidence is $19 \%$ at 40 weeks and $42 \%$ at 42 weeks. According to Steer et al, incidence of passage of meconium during labour increases with gestational age and reaches up to $30 \%$ at 40 weeks and $50 \%$ at 42 weeks $^{16}$. These results are comparable to this study in which passage of meconium was found in $20 \%$ of all deliveries. Also, at 42 weeks, $62.5 \%$ patients had meconium in liquor.

Meconium aspiration occurs in upto $35 \%$ live births with meconium stained liquor with a range from $21 \%$ to $58 \%{ }^{17}$. Meconium aspiration syndrome causes or contributes to neonatal death in approximately $10 \%$ babies who aspirate $\mathrm{it}^{18}$. This is comparable to my study in which meconium aspiration was found in $50 \%$ of the deliveries with meconium stained liquor i-e $10 \%$ of all deliveries. Only one baby developed meconium aspiration syndrome and died which is $0.5 \%$ of all deliveries and $5 \%$ of the babies who aspirate.

The results of this study were compared with the outcome of uncomplicated singleton pregnancies from 40 to 42 weeks at Parkland Hospital, USA between 1993 to $1994^{19}$. Patients included in the study were 156,66 . Out of these , $10.5 \%$ were induced. There were 16 stillbirths and 2 neonatal deaths. Perinatal mortality as 3.5/1000 live births. This study showed overall induction rate as $9.5 \%$ there was no still birth and only one neonatal death. Perinatal mortality was 5/1000.

Platt and coworkers found that fetal surveillance was clearly beneficial because fetal mortality was significantly less in closely monitored pregnancies ${ }^{20}$. Thacker and Berkelman reviewed 600 reports and found only 4 randomized controlled trials performed with non stress test. The number of these trials were considered to be too small to detect important benefits and they did not support the benefit of either test ${ }^{21}$.

Mohide and Keirse reviewed controlled clinical trials concerning antepartum fetal surveillance; they found that studies in which managements with and without fetal tests were compared, revealed no benefit ${ }^{22}$ of one over other. In a recent randomized controlled trial, there is also no difference between induced and monitored groups (every three days) in neonatal morbidity, mode of delivery and general outcome ${ }^{23}$.

Erkin and co authors described antepartum tests to be form of care likely to be ineffective ${ }^{24}$. The results of this study have shown that monitoring of the fetus with biophysical profile and non stress test between 40 to 42 weeks had no benefit over monitoring the fetus with clinical assessment and fetal kick count chart. These results are comparable to the results found in above mentioned studies ${ }^{23,24}$.

\section{CONCLUSIONS}

Exact and careful assessment of gestational age will help to reduce the overall incidence of ongoing pregnancies beyond 40 weeks. As $66 \%$ of primigravidae deliver after expected date of delivery and $10 \%$ of all pregnant women after 42 weeks of gestation: hence an exact date of delivery cannot be given.

After 40 weeks of gestation, patient should be monitored carefully with proper counseling and fetal kick chart. Conservative management with selective fetal surveillance is recommended as risks of fetal morbidity and mortality increase after 40 weeks. The methods of fetal surveillance have not been perfected so the baby should be delivered by 42 weeks irrespective of the condition of cervix.

\section{REFERENCES}

1. Harrington DJ, Mackenzie IZ, Thompson K,et al. Does a first trimester dating scan using crown rumplength reduce the risk of induction of labour for prolonged pregnancy? BJOG 2006;113(2):171-176.

2. Guidetti DA, Divan MY, Langer O. Postdate fetal surveillance; is 41 weeks too early? Am J Obstet Gynecol 1989;161:91-3. 
3. Fonseca, Linda MD,Manju MD. Am J Obstet Gynecol 2003;188(5);1214-16.

4. Grant JM. Induction of labour confers benefits in prolonged pregnancy. Br J Obtet Gynecol.1994;101:99109.

5. Grubb DK, Rabella YA, Paul RH. Post term pregnancy fetal death rate with antepartum surveillance. Obstetrics and Gynecology.1992;79:1024-1026.

6. Ehrenstein V, Pederson L, Holsteen V,et al. Post term delivery and risk for epilepsy in childhood. Pediatrics 2007;119 (3):554-561.

7. Royal college of gynecologists and obstetricians. Induction of labour. Evidence based clinical guideline. London, RCOG Press 2001:24-26.

8. Bakketeig $L$, Bergsjo P. Post term pregnancy: magnitude of problems. Chalmers I,Enkin M, Keirse MJNC(eds).Effective care in pregnancy and childbirth, vol 1,Oxford university Press 1991:765-775.

9. Hall Mh, Carre Hill RA, Fraser C, Campbell D, Samphier $\mathrm{MI}$. The extent and antecedents of uncertain gestation. Br J Obstet gynecol 1985;92:445-451.

10. Neilson JP. Ultrasound for fetal assessment in early pregnancy. Cochrane Database Syst Review 2004(3): CD 000182.

11. Reuss MI, Hatch MC, Susser M. Early ultrasound dating of pregnancy selection and measurement biases. $J$ Clin Epidemol; 48:667.

12. Lindell A. Prolonged pregnancy. Acta obstetrician et Gynecologia Scandanavia 1956; 35:136-163.

13. Rane SM, Guirgis RR, Higgins B, Nicolaides $\mathrm{KH}$. Preinduction sonographic measurement of cervical length in prolonged pregnancy. Ultrasound in Obs \& Gyne 2003;22:45-48.

14. James M, Alexander MD, Donald D. Induction of labour births. Obst \& Gynecol 2001;97:911-915.
15. The national institute of child health and human development network of maternal fetal medicine unit. A clinical trial of induction of labour versus expectant management in post term pregnancy. Am J Obstet Gynecol 1994;170:716-723

16. Steer Pj, Eigbe F, Issauer TJ. Interrelationships among abnormal cardiatocograms in labour, meconium staining of amniotic fluid, arterial cord blood PH and APGAR scorel.Obstetrics and Gynecology;1989.74:715721.

17. Gregory GA, Gooding CA, Phibbs RH, Tooley WH. Meconium aspirations in infants-a prospective study. Journal of paediatrics ;1974.85:848-852.

18. Wiswell TE, Tuggle JM, Turner BS. Meconium aspiration syndrome, have we made a difference. Paediatrics;1990.85:715-721.

19. Cunningham FG,Mac Donald,Leveno KJ et al.Williams Obstetrics, $20^{\text {th }}$ edition .Appleton and Lange, Stanford Connecticut;1997:827-837.

20. Platt LD, Paul RH, Phelan J, walla CA, Broussard P. Fifteem years of experience with antepartum fetal testing. Am J Obstet Gynecol;1987.156:1509.

21. Thacker SB, Berkelman RL. Assessing the diagnostic accuracy and efficiency of selected antepartumfetal surveillance techniques. Obstet gynecol Surv 1986;41:121.

22. Mohide $P$, Keirse MJNC. Biophysical assessment of fetal well being. In Chambers I, Erkin M, Keirse MJNC(eds);Effective care pregnancy and childbirth, vol l; Pregnancy. Newyork. Oxford University Press;1999:477.

23. Heimstad R, Skogvoll E, Maltson LA, et al. Induction of labour versus serial antepartum fetal monitoring in post term pregnancy. Obstet \& Gynecol 2007;109(3):609-617.

24. Erkin M, Keirse MJNC, Renfrew M, Neilson. A guide to effective care $\mathrm{n}$ pregnancy and child birth, $2^{\text {nd }}$ edition ,New York, Oxford University press;1995:410. 